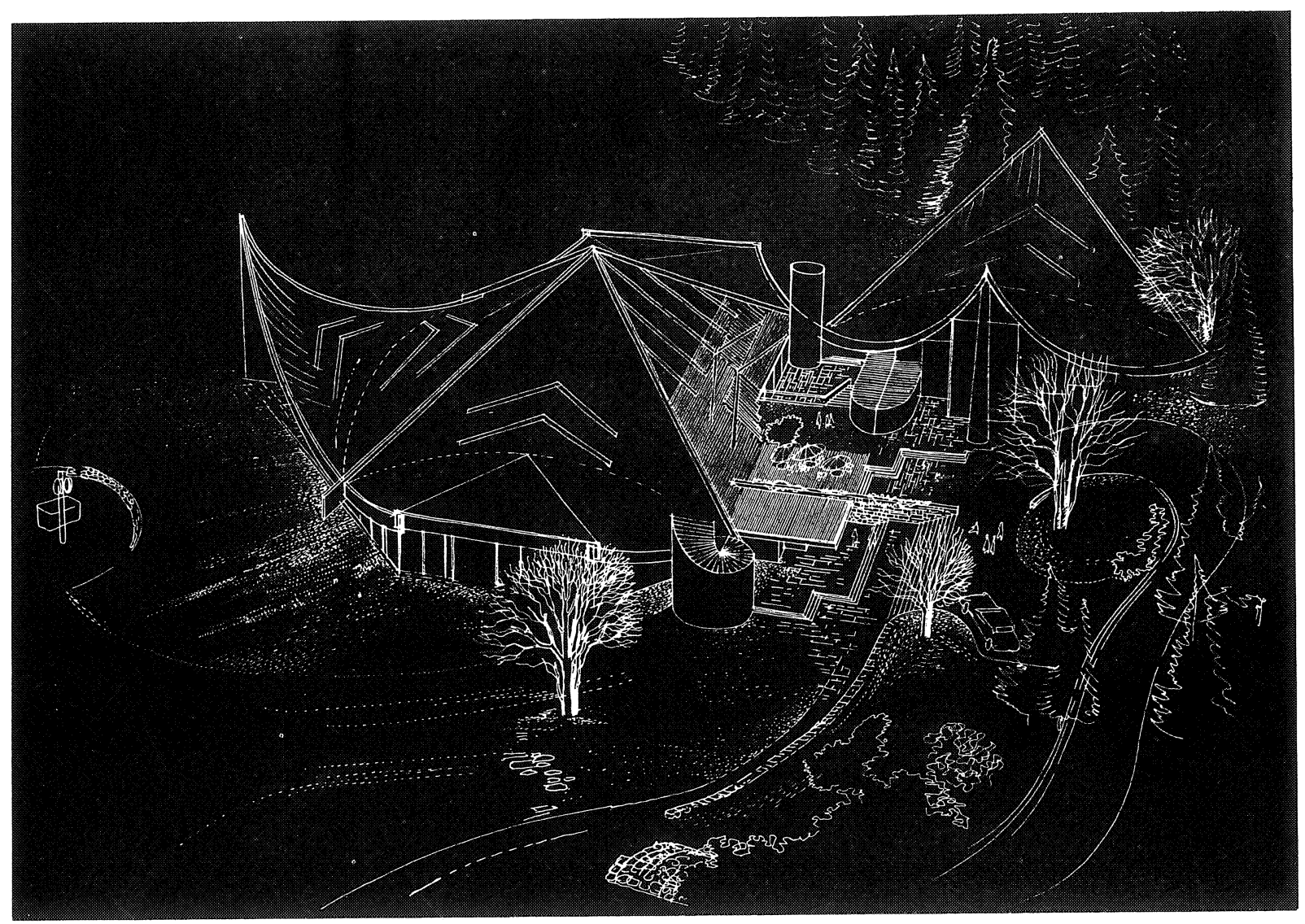

\title{
sala polideportiva de Super-Lioran \\ Francia
}

Vittorio Mazzucconi, arquitecto Jean Marie F. Hereng, ingeniero

$158-12$

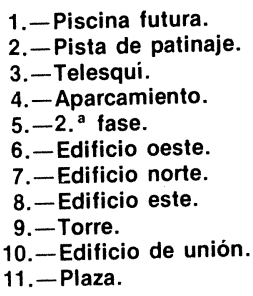

\section{situación}
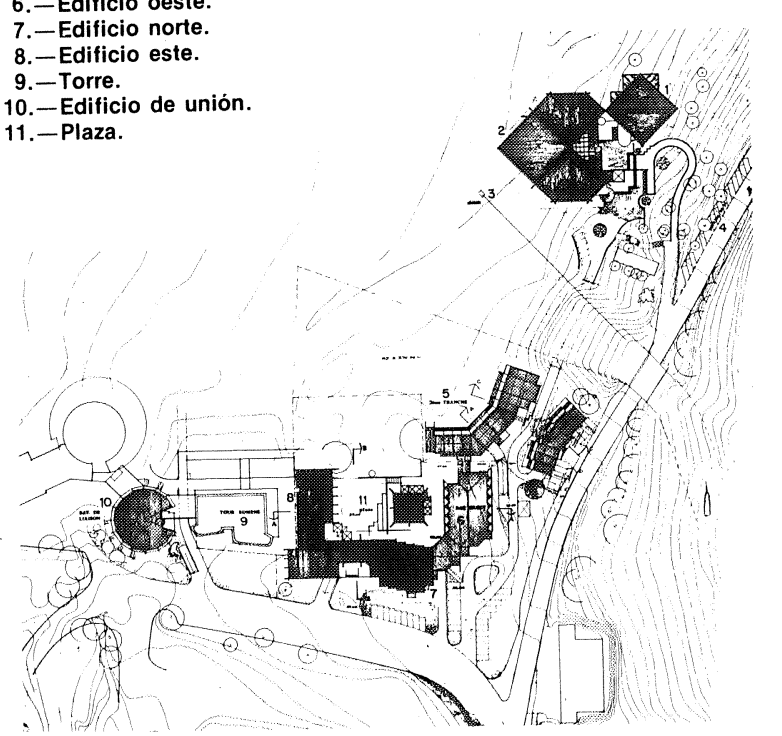

\section{sinopsis}

La sala polideportiva de la estación invernal de Super-Lioran, situada en un cráter relleno de depósitos aluvionares, consta de dos partes claramente diferenciadas: una inferior, de hormigón, donde se encuentran la pista de patinaje y los distintos servicios, y una superior formada por elementos de paraboloide hiperbólico. Esta cubierta es de madera laminada encolada.

La estructura laminar de la cubierta se apoya en el suelo casi confundièndose con él, y luego emerge desde la nieve proporcionando al conjunto un carácter casi abstracto que contribuye a aumentar la belleza de la zona. 
La Sala forma parte de un conjunto proyectado para la estación de invierno de Super-Lioran.

El emplazamiento se encuentra en un lugar magnífico, conocido por "Pradera de Sagnes", en el cráter del volcán de Cantal. Esta zona está rellena de depósitos de aluvión procedentes de las laderas próximas.

La belleza del lugar, la necesidad de no chocar con

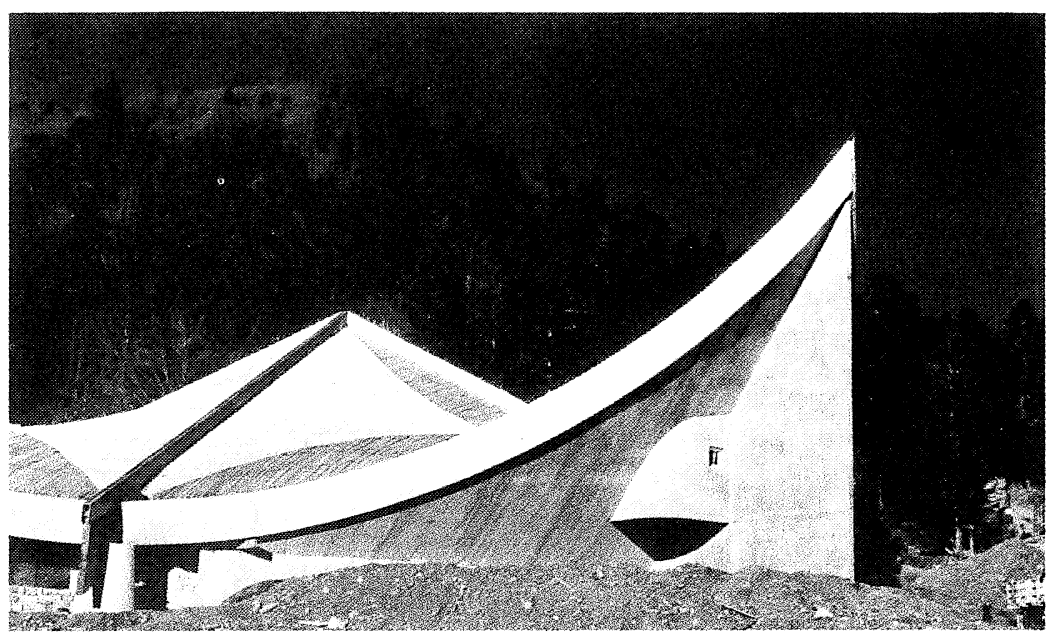

vista parcial (fachada sur)

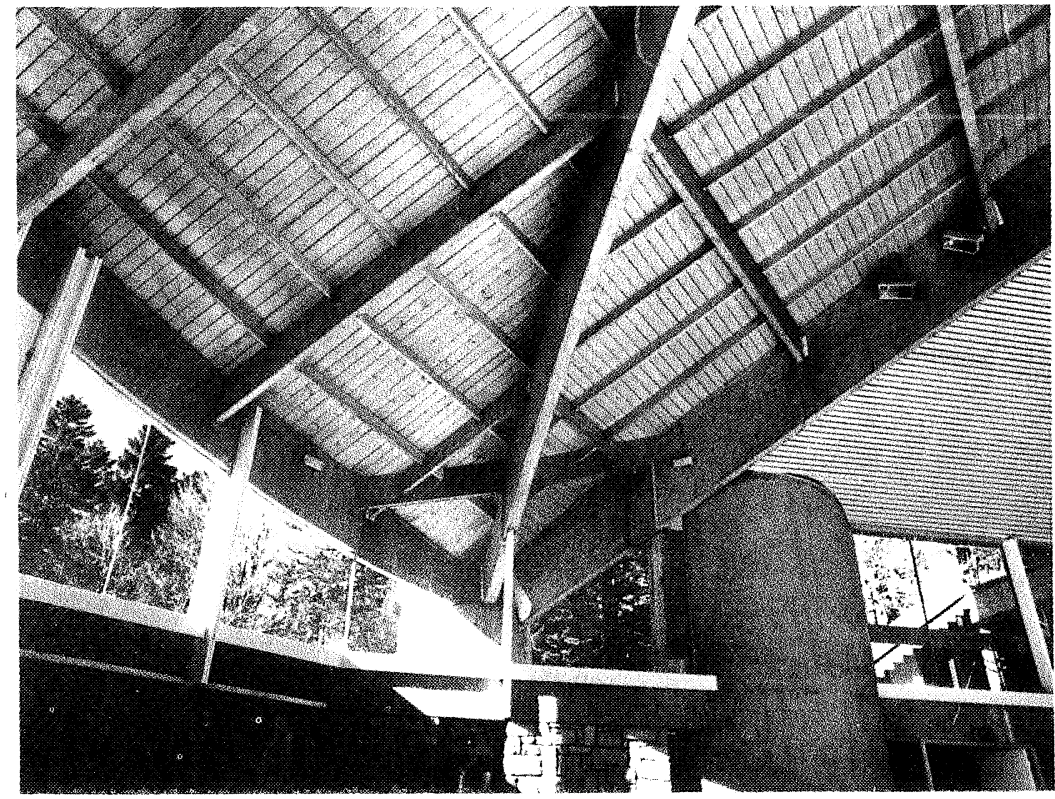

detalle de uno de los cuatro puntos de apoyo

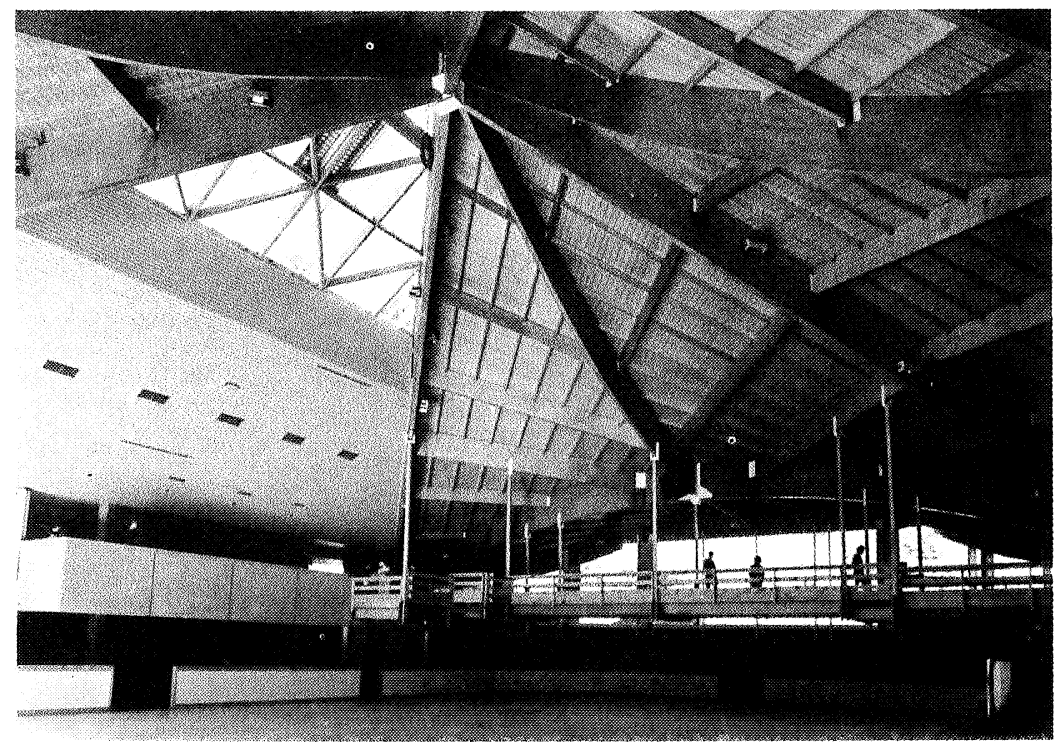

vista interior hacia la pasarela las tradiciones populares e, incluso, el hecho de que la Sala debiera erigirse sobre un volcán, lo que le da un cierto aspecto misterioso $y$, si se quiere, mágico, influyeron notablemente en el proyectista a la hora de buscar una solución idónea.

El plan urbanístico de la zona conectando con la tradición regional, del Auvergne, previó la construcción, en un rincón de la pradera, de un conjunto de edificios para viviendas y usos comerciales, como si se tratase de un pueblo. Por otro lado, al igual que algunas ciudades costeras italianas que tienen una estructura urbanística en forma de plaza abierta hacia el mar, aquí se consideró a la pradera como una especie de mar imaginario y se construyó un gran barco que la surca.

Este barco es la Sala de deportes y recreo de la estación de invierno de Super Lioran.

Posteriormente se construirá una segunda sala más pequeña para piscina cubierta. 


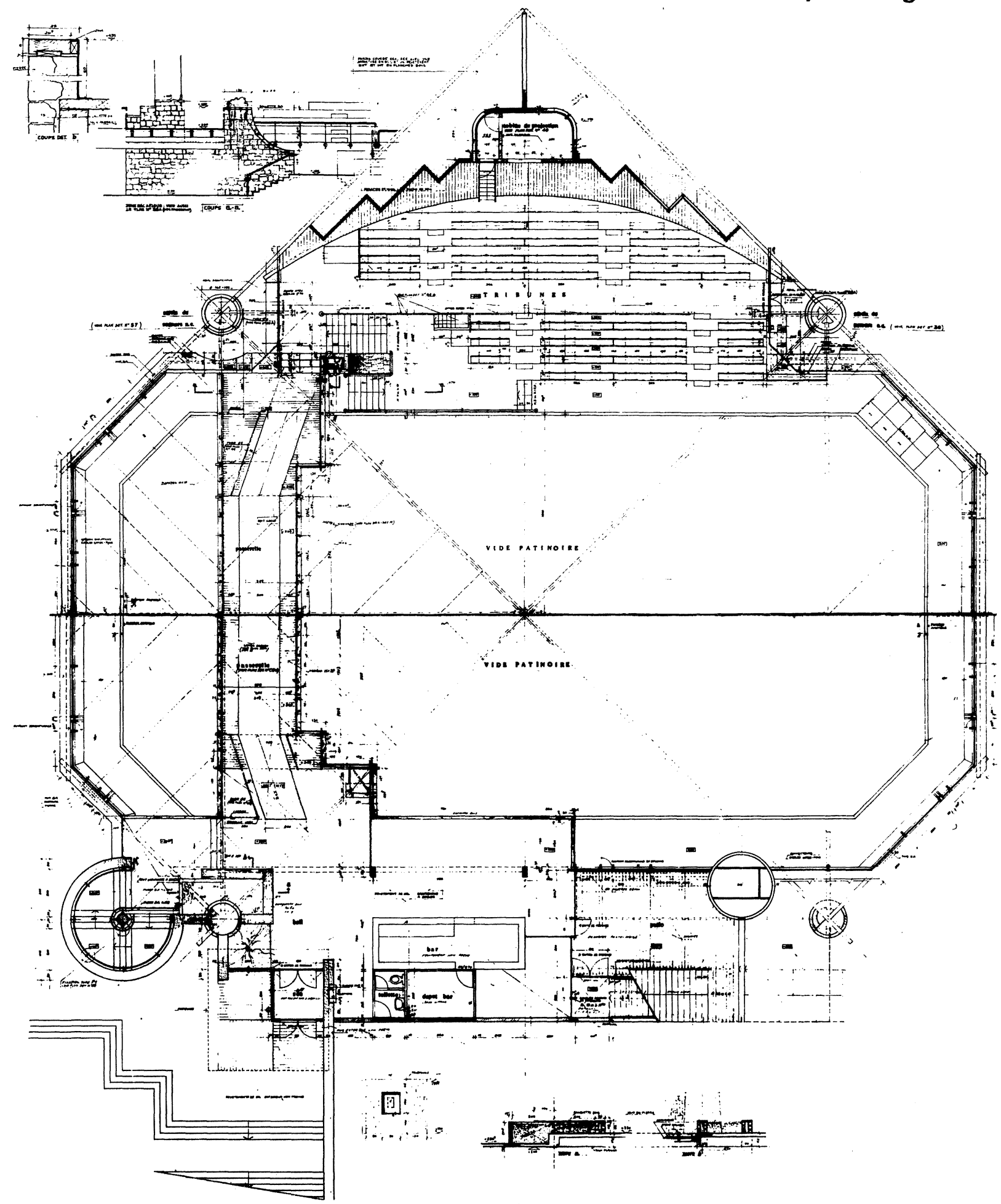

La Sala polideportiva tiene dos partes perfectamente diferenciadas: la inferior, de hormigón armado y pretensado, ligeramente eriterrada que soporta la pista de patinaje propiamente dicha, los servicios, la maquinaria y el almacén de material, el bar, los vestuarios y lavabos, el hall de entrada y las tribunas; y la superior, que constituye la cubierta y que abarca todo el conjunto, formada por elementos de paraboloide hiperbólico unidos. Esta cubierta sustentada por cuatro puntos de apoyo es de madera laminada encolada.

Precisamente el hecho de que la Sala esté en parte enterrada en el terreno hace que al entrar en ella, viniendo desde la parte exterior, nevada, se tenga una impresión muy cálida, como si se 
entrase en la bodega de un barco.

El conjunto de la obra se sustenta en pilotes hincados en el suelo hasta la capa de basalto. Las cargas se transmiten a los pilotes por medio de un entramado de vigas riostras de hormigón armado. En aquellos
Los cuatro apoyos aseguran el equilibrio de la estructura y se calcularon para transmitir a los pilotes solamente los esfuerzos verticales positivos. La estabilidad horizontal se asegura mediante tirantes pretensados. Estos en una de las dos direcciones van integrados en el solado $y$ en el forjado, mado y aseguran la contención del terreno. Los tabiques interiores, bajo las tribunas o locales de servicios, son mixtos: contravientos de hormigón armado y las demás partes de bloques de hormigón.

La pared circular de la torre en la que está situada la

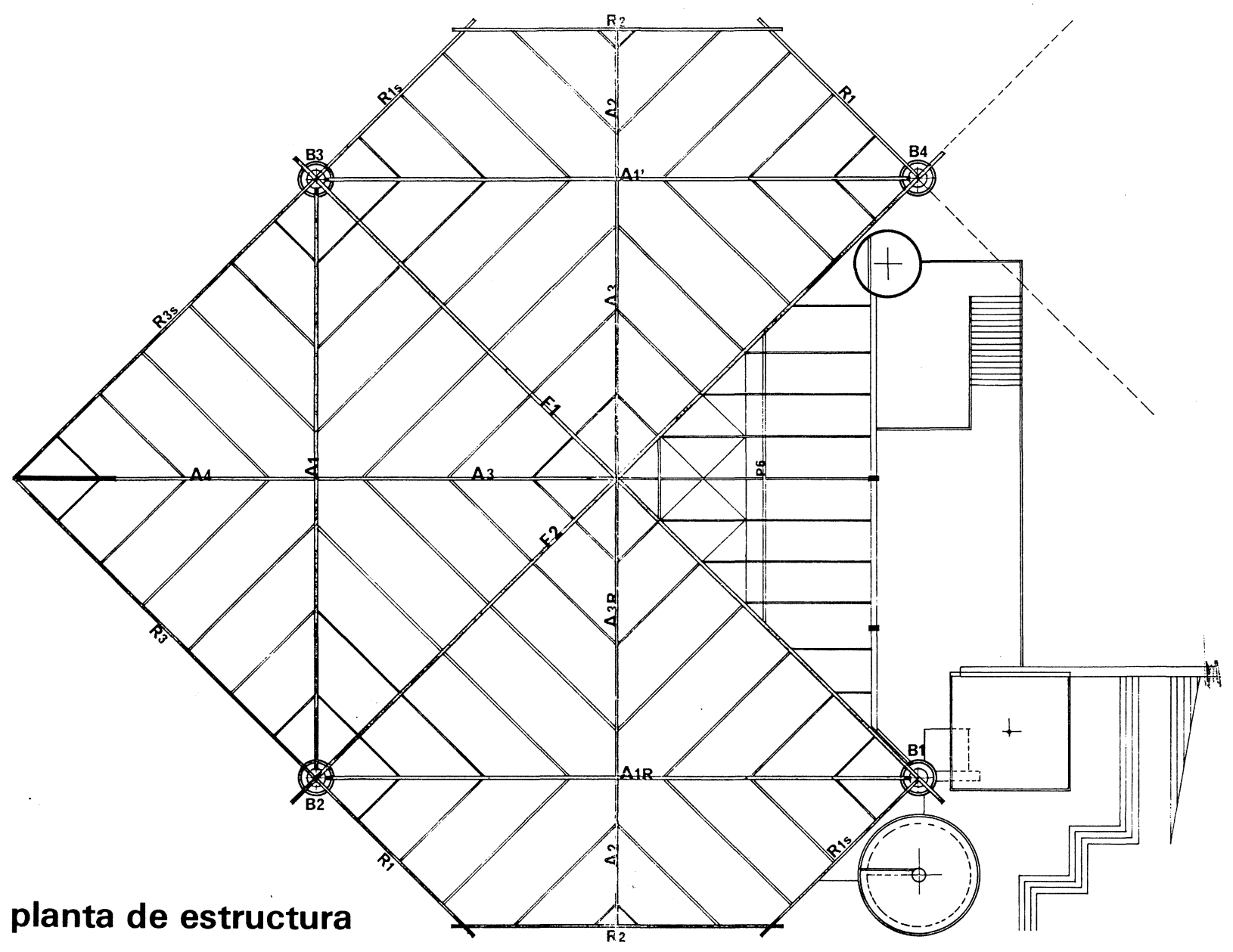

casos en que es necesaria una gran rigidez, estas vigas se unen a las láminas de hormigón armado situadas sobre ellas.

Para evitar deformaciones, la pista de patinaje propiamente dicha, que lleva su propio entramado, está separada del resto de la obra por una junta de dilatación periférica. mientras que en la dirección perpendicular se encuentran suspendidos de las vigas riostras.

Las losas situadas sobre los sanitarios y el pavimento realizado sobre el relleno se calcularon como losas bidireccionales continuas.

Los muros periféricos exteriores son de hormigón ar- escalera principal por la que se accede a la planta inferior se realizó con bloques de piedra vista de $50 \mathrm{~cm}$ de espesor.

La chimenea, de sección circular para entrada y salida del aire, así como para la calefacción, es de hormigón armado desencofrado y sin posterior acabado. 

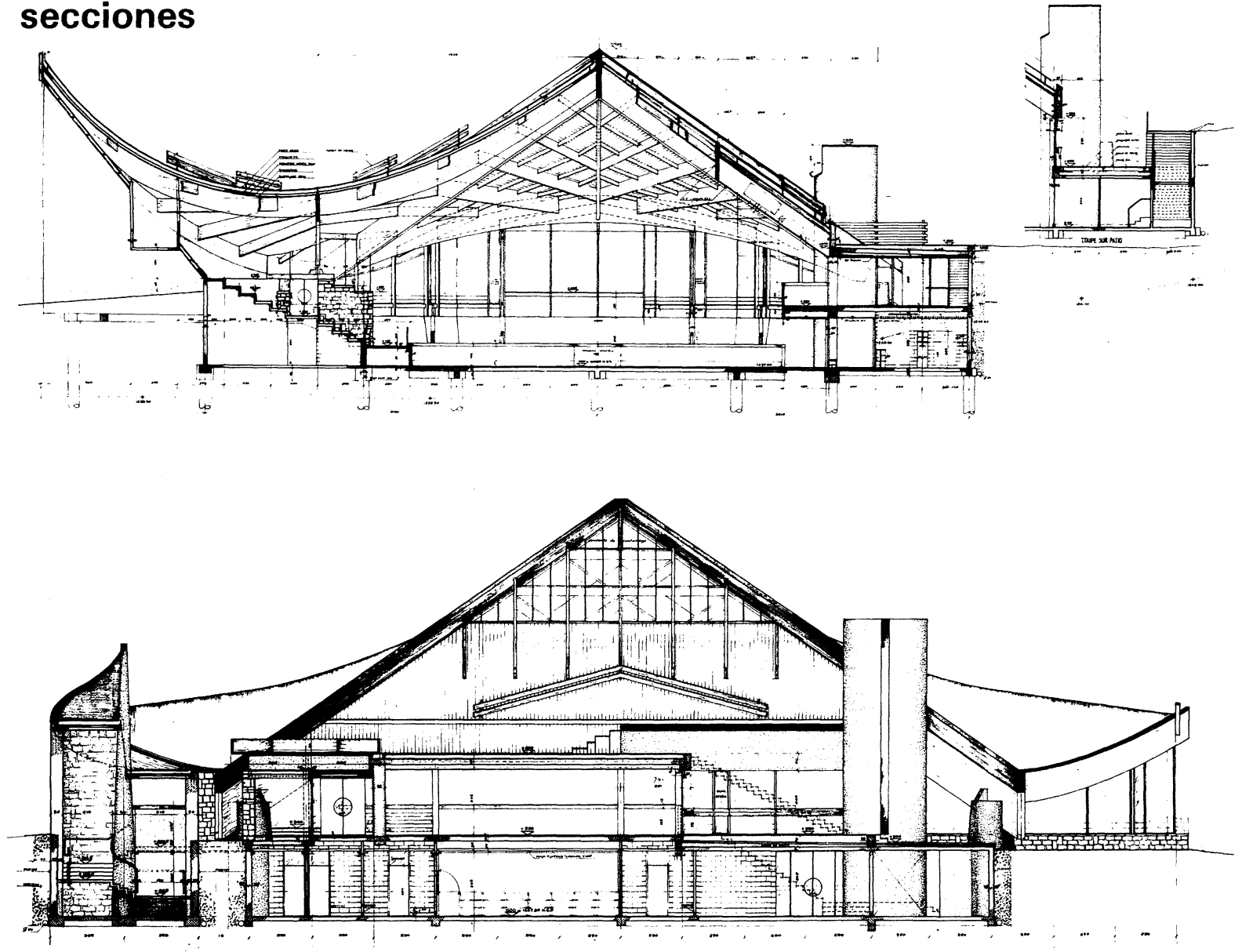

El forjado inferior de la pista de patinaje se realizó mediante losas alveoladas pretensadas prefabricadas.

El forjado superior situado sobre los locales de servicio y técnicos, asi como el situado sobre la entrada, bar y vestuarios es de hormigón armado formado por losas en dos direcciones apoyadas sobre vigas.

Asimismo, tanto las gradas de las tribunas como la marquesina situada sobre la entrada principal están hechas de hormigón armado: las gradas, con elementos prefabricados y la marquesina como una losa simple sin vigas apoyada en un solo pilar metálico.

La escalera exterior entre la terraza y la futura piscina tiene un apoyo central y los escalones en ménsula a ambos lados.

Las escaleras interiores son de hormigón armado colado, teniendo la escalera principal 17 escalones con descansillos cada cuatro escalones, o sea, cada cuarto de circular.

La cubierta de esta torre de la escalera principal es una lámina helicoidal de hormigón armado.

La estructura de la cubierta, que emerge del suelo y le da un aspecto abstracto a todo el conjunto está constituida, como ya se ha dicho, por elementos de paraboloides hiperbólicos unidos de madera laminada encolada.
Una de las mayores dificultades que hubo que resolver estribó en que los bordes exteriores, rectos inicialmente, se tenían que transformar, por razones arquitectónicas evidentes, en parábolas. Debido a esto, la superficie no era desarrollable sino una superficie de curvatura variable, positiva en una parte y negativa en otra con los puntos de inflexión situados sobre la hipérbola cóncava que forma una de las diagonales.

La solución adoptada permitió realizar esta superficie, teóricamente no desarrollable, mediante elementos totalmente rectos con algunos elementos curvos: las diagonales interiores que tienen forma parabólica e hiperbólica y las vigas de borde exteriores de forma para- 

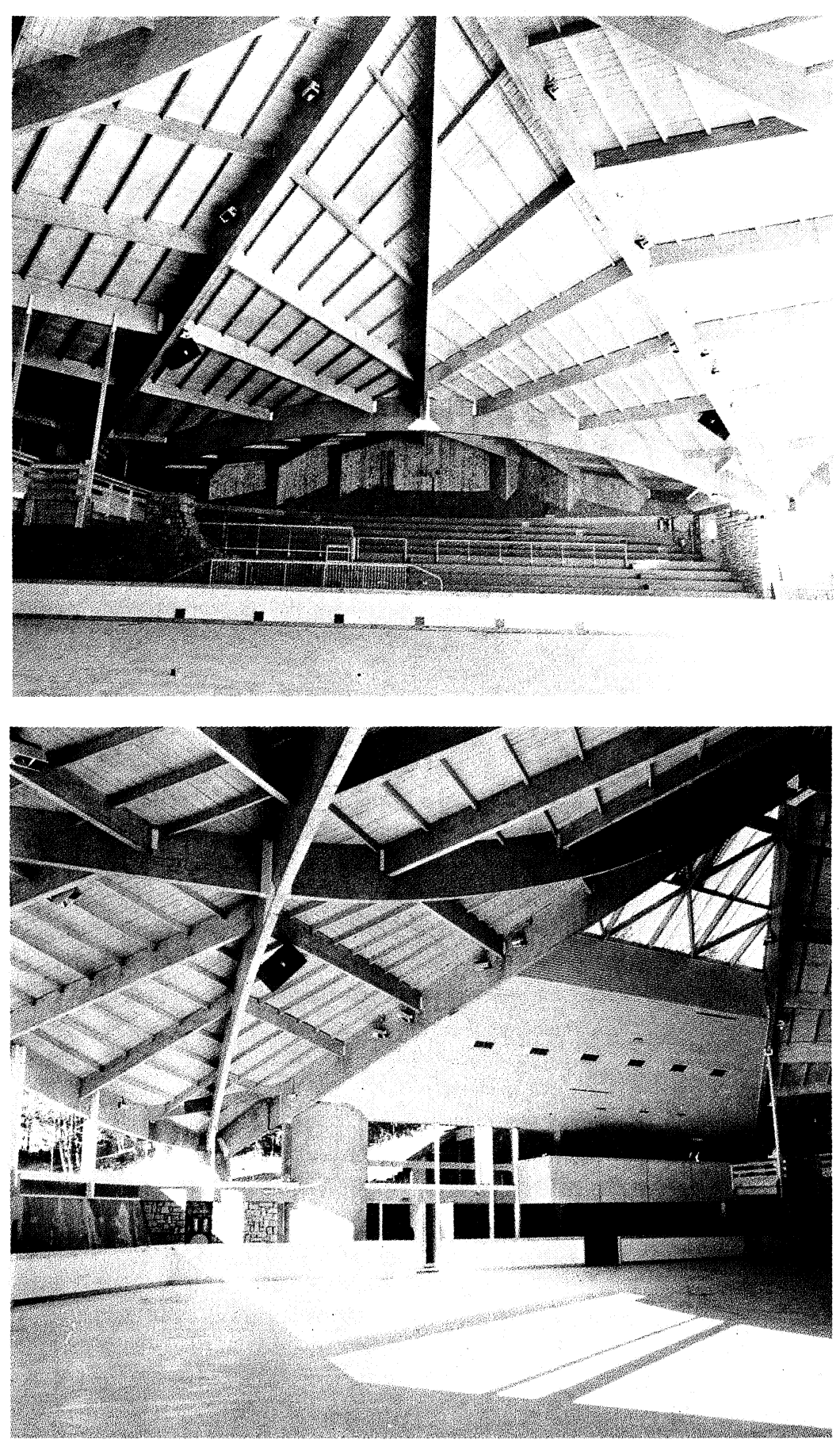

résumé

SALLE POLYVALENTE DE SPORTS DU SUPER LIORAN (CANTAL) FRANCE

Vittorio Mazzucconi, architecte Jean-Marie Hereng, ingénieur structures

La salle polyvalente de sports de la station de sports d'hiver du Super Lioran, située sur un crátère comblé par les dépôts alluvionnaires, a été réalisée en deux parties: la première, en béton, comporte la patinoire et les différents services, et la seconde est constituée par des éléments de paraboloïdes hyperboliques. Cette couverture est en bois lamellé collé.

La couverture s'appuie au sol avec presque une volonté de se confondre avec la terre et pues, elle émerge de la neige, conférant à l'ensemble un caractère presque abstrait qui contribue à augmenter la beauté de la zone. summary

\section{vistas interiores}

bólica. Esta original solución permitió reducir el coste de la estructura a la mitad.

Otra solución bastante original fue la articulación entre los pórticos de $43 \mathrm{~m}$ de luz y los arcos parabólicos Estas articulaciones están hechas con placas de apoyo de neopreno zunchado interpuestas entre las zapatas metálicas del extremo de las piezas de la estructura y el hormigón de los puntos de apoyo.

La, cubierta propiamente dicha está formada por un entarimado machiembrado de madera de friso que soporta el aislamiento térmico y una cámara de estanquidad a base de elastómeros $y$, por encima de ella, una estructura para nieve de madera de alerce vista, en láminas separadas.

La pasarela que une los servicios de la entrada principal con las tribunas, y que permite al público una panorámica de la sala y de su ambiente, está constituida igualmente por una estructura laminada encolada. Está suspendida de las vigas mestras de la estructura principal.

\section{zusammenfassung}

SUPER-LIORAN POLY-SPORTS HALL FRANCE

Vittorio Mazzucconi, architect Jean Marie F. Hereng, engineer (HISA. CORP)

The polysports hall of the Super-Lioran winter resort, located in a crater filled with alluvionary deposits, consists of two clearly differentiated is the skating rink and various services, and an upper one consisting of hyperbolic paraboloid elements. This roof is of adhered laminate wood

The laminate roof structure rests on the ground, and almost merges with it, to later emerge from the snow giving the assembly almost an abstract nature which helps to almost an abstract nature whic increase the beauty of the area.

\section{SPORTHALLE IN SUPER-LIORAN - FRANKREICH}

Architekt: Vittorio Mazzucconi

Ingenieur: Jean Marie F. Hereng (HISA, CORP)

Die Sporthalle der Ski-Station Super-Lioran welche sich in einem mit Wasserflutablage rungen gefüllten Krater befindet, besteht aus zwei klar unterschiedenen Teilen: einem unteren Betonteil, welcher die Eisbahn und verschieAnlagen beherbergt, und dem oberen Teil, der aus hyperbolischen Paraboloiden gebildet wird. Das Dach besteht aus ver leimten Holzlamellen.

Die Lamellenstruktur des Daches stützt sich derartig auf dem Boden ab, dass sie fast in diesen übergeht, um später aus dem Schnee Komplex einen fast abstrakten Charakter Komplex einen fast abstrakten Charakter
verleiht, der dazu beiträgt, dises Gebiet noch schöner zu gestalten. hervorzuragen, wodurch sie dem gesamten 